\section{Case Reports in Oncology}

Case Rep Oncol 2018;11:281-288

DOl: $10.1159 / 000489085$

Published online: May 17, 2018

(C) 2018 The Author(s)

Published by S. Karger AG, Base

www.karger.com/cro

This article is licensed under the Creative Commons Attribution-NonCommercial 4.0 International License (CC BY-NC) (http://www.karger.com/Services/OpenAccessLicense).

Usage and distribution for commercial purposes requires written permission.

\title{
Treatment of Leptomeningeal Carcinomatosis in a Patient with Metastatic Pancreatic Cancer: A Case Report and Review of the Literature
}

\author{
William Rainey Johnson Brett J. Theeler David Van Echo \\ Patrick Young Mary Kwok \\ Walter Reed National Military Medical Center, Bethesda, MD, USA
}

\section{Keywords}

Leptomeningeal disease - Brain metastasis - Intrathecal chemotherapy · Pancreatic cancer . FOLFIRI $\cdot$ FOLFIRINOX $\cdot$ Neoplastic meningitis

\begin{abstract}
Pancreatic cancer is the fourth leading cause of cancer-related death with a median survival of 3-11 months when metastatic. We present a patient with metastatic pancreatic cancer and an exceptional response to initial systemic chemotherapy with FOLFIRINOX (fluorouracil, leucovorin, irinotecan, and oxaliplatin). Despite evidence of disease control on body imaging, he developed symptomatic leptomeningeal disease and brain metastases 29 months into treatment. He received aggressive treatment with capecitabine and irinotecan, intrathecal topotecan, and eventually bevacizumab. He did well for 36 weeks on this regimen until developing sepsis. This patient significantly outlived his expected survival and, moreover, did so with very good quality of life. This case demonstrates the natural history of pancreatic cancer progressing to involve the central nervous system when systemic disease is otherwise responsive to chemotherapy. It is the first case to demonstrate the potential effectiveness of intrathecal topotecan in combination with systemic chemotherapy for the treatment of leptomeningeal metastases of pancreatic cancer.




\section{Introduction}

Pancreatic adenocarcinoma is the fourth leading cause of cancer-related death in the United States, with an estimated 40,560 deaths in 2015 alone [1]. The overall 5-year survival is less than $5 \%$, with a median survival of 3.5 months for untreated advanced disease. Moreover, fewer than $20 \%$ of patients present with localized, potentially curable, pancreatic tumors $[1,2]$. The most common sites of metastatic disease are the liver and peritoneal cavity $[2,3]$. Metastases to the central nervous system (CNS) are exceedingly rare, with leptomeningeal metastases representing an even smaller subset of CNS metastases [4]. In a search of PubMed, EMBASE, and Scopus, we found only 18 cases of pancreatic leptomeningeal metastasis from 1975 to October 2015. The longest survival documented in the English literature was 6 months following identification of leptomeningeal metastases from pancreatic cancer in a patient treated with intrathecal methotrexate and radiation. We report a case of a patient with metastatic pancreatic cancer, who had an exceptional response to initial standard therapy before developing leptomeningeal disease after 29 months, which responded well to the addition of intrathecal therapy for 9 months - three times the median expected survival.

\section{Case Report}

A 53-year-old man presented to the emergency department with 3 weeks of loose acholic stool, epigastric pain, and weight loss. He had a bilirubin level of $9.0 \mathrm{mg} / \mathrm{dL}$, elevated liver enzymes, and elevated CA 19-9 of 22,700 units/mL. CT imaging demonstrated multiple liver lesions and dilation of the common bile duct. A liver biopsy revealed carcinoma that was positive for CK7 but negative for CK20, CEA, and TTF-1, consistent with carcinoma of pancreaticobiliary origin. A repeat CT scan showed interval growth of too-numerous-to-count liver masses and a hypodense lesion arising from the uncinate process of the pancreas (Fig. 1). Endoscopic ultrasound with fine-needle aspiration of the pancreatic lesion was diagnostic for pancreatic adenocarcinoma. The patient began therapy with FOLFIRINOX (fluorouracil, leucovorin, irinotecan, and oxaliplatin) chemotherapy every 2 weeks. His course was complicated by thrombocytopenia, leading to dose reduction, and distal neuropathy, leading to discontinuation of oxaliplatin after cycle $23[5,6]$. He continued with FOLFIRI and had a good clinical response with falling CA 19-9 levels and radiographic stabilization or reduction of masses during treatment (Fig. 2). He regained pancreatic function and returned to an excellent quality of life, resuming his work in carpentry and participating in recreational activities. After 29 months (63 cycles), he complained of intermittent throbbing occipital headaches, dysarthria, and word-finding difficulty. A brain MRI demonstrated intracranial and leptomeningeal metastasis, with drop metastases from T10 to the conus medullaris (Fig. 3), despite control of systemic disease (Fig. 1). He received whole-brain radiation therapy and subsequently began intrathecal topotecan via an Ommaya reservoir, with an induction phase of $0.4 \mathrm{mg}$ twice weekly for 4 weeks and consolidation phase of $0.4 \mathrm{mg}$ weekly thereafter. His systemic chemotherapy was changed to capecitabine and irinotecan (CAPIRI) due to improved CNS penetration of capecitabine compared with fluorouracil. His leptomeningeal disease responded radiographically, but at 35 months demonstrated radiographic progression leading to the addition of bevacizumab (Fig. 3). He continued on this regimen until 38 months from initial diagnosis. At that point, he was admitted to the hospital for altered mental status without radiographic progression of his leptomeningeal or intracranial disease. He was found to have sepsis, complicated by septic shock, disseminated intravascular coagulation, and developed a hospital- 
acquired aspiration pneumonia after endotracheal intubation. He recovered from these complications, but after 2.5 weeks without chemotherapy - held in the setting of his sepsis - he had radiographic progression of his leptomeningeal metastases and return of dysarthria and word-finding difficulties. After extensive discussion, he was discharged to home hospice and died 1 month later.

\section{Discussion}

Leptomeningeal spread of pancreatic cancer is increasingly reported in the medical literature. However, only two articles have described a treatment approach, both with limited effectiveness. Minchom et al. [7] described a patient treated with intrathecal hydrocortisone, methotrexate, and cytarabine, which were paired with systemic gemcitabine. Due to clinical deterioration, they stopped the chemotherapy after 2 weeks, and the patient expired within 6 weeks. Rebischung et al. [8] reported a 44-year-old female with pancreatic leptomeningeal metastases treated with 5-iodo-2'-deoxyuridine with 125-Iodine isotope and intrathecal methotrexate. The initial radiographic response was promising; however, there was progression of the neoplastic meningitis at 3 months, and the patient died at 6 months. To our knowledge, our case represents the first report of intrathecal topotecan for treatment of pancreatic leptomeningeal metastases. The following is a discussion of this novel treatment approach for pancreatic leptomeningeal disease.

Topotecan is a topoisomerase I inhibitor, which is approved for treatment of recurrent or resistant cervical cancer, metastatic ovarian cancer, and relapses of small cell lung cancer. Topotecan is safe in the CNS. When administered intravenously, about 30\% reaches the CNS; however, concentrations can be increased with intrathecal administration [9-11]. Blaney et al. [11] investigated appropriate intrathecal dosing and found maximum tolerable dosage of $0.4 \mathrm{mg}$, with the most common side effects being nausea/vomiting, headache, anorexia, and fever. Arachnoiditis, a more significant side effect, did not affect patients in the 0.2- or 0.4-mg groups [11]. Since then, intrathecal topotecan has been used in treatment of leptomeningeal metastases from tumors arising from the CNS, breasts, ovaries, skin (melanoma), lungs, sinus, blood (multiple myeloma, leukemia), and esophagus. Groves et al. [12] investigated intrathecal topotecan in 62 patients with neoplastic meningitis from a variety of primary tumors (none of pancreatic origin) with 40 patients completing the initial 6-week induction phase. They found that $16 \%$ had clinical improvement after induction, 30\% were free of progression at 13 weeks, and $19 \%$ free of progression at 52 weeks. The mean time to progression was 17 and 6 weeks for malignancies of CNS and non-CNS origin, which is consistent with the literature $[12,13]$.

We selected intrathecal topotecan for the treatment of our patient's pancreatic leptomeningeal carcinomatosis due to this 2008 investigation demonstrating safety and possible effectiveness, and our patient's desire to explore possible treatment options despite the lack of known effective therapy [12]. Without a direct comparison of intrathecal agents available in the setting of an extraordinarily rare presentation of pancreatic cancer metastasis to the leptomeninges, we chose topotecan over methotrexate because topotecan has better long-term tolerability and is associated with a lower rate of arachnoiditis [12]. We deviated from the standard dosing slightly, decreasing the induction phase to 4 weeks and then continuing weekly thereafter to accommodate his travel and quality of life $[12,14]$. Given control of systemic disease with FOLFIRI, we paired this intrathecal regimen with CAPIRI due to capecita- 
bine's similar effectiveness to fluorouracil and better CNS penetration for intracranial parenchymal disease $[6,15]$. With the combination of intrathecal topotecan and systemic CAPIRI treatment, our patient did not have progression of his CNS disease for 35 weeks from initiation of CNS-directed treatment. At this point, we added bevacizumab based on its potential to enhance cytotoxic effects of chemotherapy in the CNS and its efficacy in other gastrointestinal malignancies [16-20]. He tolerated the initial treatment of CAPIRI and topotecan extremely well; however, after starting bevacizumab, he had some mild confusion, likely representing a grade 1-2 adverse event. Thirty-six weeks after intrathecal treatment initiation, he presented to the hospital with altered mental status. While this may have represented a grade 3 adverse event, it was more likely the patient's sepsis, secondary to pyelonephritis and emphysematous cystitis. At the time of his admission, his leptomeningeal disease and brain metastases were stable from 3 months prior, demonstrating the effectiveness of his regimen. After recovery from sepsis and progression of his leptomeningeal disease, our patient had very poor functional status, guiding the decision for palliative care and hospice.

Extremely few cases exist to guide the therapy of pancreatic leptomeningeal carcinomatosis; however, with the improvement in cancer treatments and survival, the incidence of this complication is likely to increase. The patient tolerated the combination of CAPIRI, bevacizumab, and intrathecal topotecan well, and it was effective in stabilizing his leptomeningeal disease and brain metastases. Our patient outlived his expected survival in the setting of leptomeningeal metastasis and did so with a reasonable quality of life. Combinatorial treatment including CAPIRI, intrathecal topotecan, plus or minus bevacizumab may warrant further evaluation in pancreatic leptomeningeal carcinomatosis.

\section{Statement of Ethics}

The patient gave consent for the publication of his case.

\section{Disclosure Statement}

The authors declare no competing financial interest and no conflicts of interest. The views expressed in this paper are those of the author and do not reflect the official policy of the Department of Army, Navy, Air Force, Department of Defense, or US Government. The identification of specific products, scientific instrumentation, or organizations is considered an integral part of the scientific endeavor and does not constitute endorsement or implied endorsement on the part of the author, DoD, or any component agency.

\section{References}

Siegel RL, Miller KD, Jemal A. Cancer statistics, 2015. CA Cancer J Clin. 2015 Jan-Feb;65(1):5-29.

2 Hidalgo M. Pancreatic cancer. N Engl J Med. 2010 Apr;362(17):1605-17.

3 Yachida S, Iacobuzio-Donahue CA. The pathology and genetics of metastatic pancreatic cancer. Arch Pathol Lab Med. 2009 Mar;133(3):413-22.

4 Lemke J, Scheele J, Kapapa T, Wirtz CR, Henne-Bruns D, Kornmann M. Brain metastasis in pancreatic cancer. Int J Mol Sci. 2013 Feb;14(2):4163-73.

5 Conroy T, Desseigne F, Ychou M, Bouché O, Guimbaud R, Bécouarn Y et al; Groupe Tumeurs Digestives of Unicancer; PRODIGE Intergroup. FOLFIRINOX versus gemcitabine for metastatic pancreatic cancer. N Engl J Med. 2011 May;364(19):1817-25. 
6 Tempero MA, Arnoletti JP, Behrman SW, Ben-Josef E, Benson AB 3rd, Casper ES et al; National Comprehensive Cancer Networks. Pancreatic Adenocarcinoma, version 2.2012: featured updates to the NCCN Guidelines. J Natl Compr Canc Netw. 2012 Jun;10(6):703-13.

7 Minchom A, Chan S, Melia W, Shah R. An unusual case of pancreatic cancer with leptomeningeal infiltration. J Gastrointest Cancer. 2010 Jun;41(2):107-9.

8 Rebischung C, Hoffmann D, Stefani L, Desruet MD, Wang K, Adelstein SJ et al. First human treatment of resistant neoplastic meningitis by intrathecal administration of MTX plus (125)IUdR. Int J Radiat Biol. 2008 Dec;84(12):1123-9.

9 Sung C, Blaney SM, Cole DE, Balis FM, Dedrick RL. A pharmacokinetic model of topotecan clearance from plasma and cerebrospinal fluid. Cancer Res. 1994 Oct;54(19):5118-22.

10 Blaney SM, Cole DE, Godwin K, Sung C, Poplack DG, Balis FM. Intrathecal administration of topotecan in nonhuman primates. Cancer Chemother Pharmacol. 1995;36(2):121-4.

11 Blaney SM, Heideman R, Berg S, Adamson P, Gillespie A, Geyer JR et al. Phase I clinical trial of intrathecal topotecan in patients with neoplastic meningitis. J Clin Oncol. 2003 Jan;21(1):143-7.

12 Groves MD, Glantz MJ, Chamberlain MC, Baumgartner KE, Conrad CA, Hsu S et al. A multicenter phase II trial of intrathecal topotecan in patients with meningeal malignancies. Neuro-oncol. 2008 Apr;10(2):208-15.

13 Gammon DC, Bhatt MS, Tran L, Van Horn A, Benvenuti M, Glantz MJ. Intrathecal topotecan in adult patients with neoplastic meningitis. Am J Health Syst Pharm. 2006 Nov 1;63(21):2083-6.

14 Nagpal S, Riess J, Wakelee H. Treatment of leptomeningeal spread of NSCLC: a continuing challenge. Curr Treat Options Oncol. 2012 Dec;13(4):491-504.

15 Stapleton SL, Reid JM, Thompson PA, Ames MM, McGovern RM, McGuffey L et al. Plasma and cerebrospinal fluid pharmacokinetics of pemetrexed after intravenous administration in non-human primates. Cancer Chemother Pharmacol. 2007 Mar;59(4):461-6.

16 Brastianos PK, Brastianos HC, Hsu W et al. The toxicity of intrathecal bevacizumab in a rabbit model of leptomeningeal carcinomatosis. J Neurooncol. 2012 Jan;106(1):81-8.

17 Chen IC, Lin CH, Jan IS, Cheng AL, Lu YS. Bevacizumab might potentiate the chemotherapeutic effect in breast cancer patients with leptomeningeal carcinomatosis. J Formos Med Assoc. 2016 Apr;115(4):243-8.

18 Souglakos J, Ziras N, Kakolyris S, Boukovinas I, Kentepozidis N, Makrantonakis P et al. Randomised phase-II trial of CAPIRI (capecitabine, irinotecan) plus bevacizumab vs FOLFIRI (folinic acid, 5 -fluorouracil, irinotecan) plus bevacizumab as first-line treatment of patients with unresectable/metastatic colorectal cancer (mCRC). Br J Cancer. 2012 Jan;106(3):453-9.

19 Hurwitz H, Fehrenbacher L, Novotny W, Cartwright T, Hainsworth J, Heim W et al. Bevacizumab plus irinotecan, fluorouracil, and leucovorin for metastatic colorectal cancer. N Engl J Med. 2004 Jun;350(23):2335-42.

20 Saltz LB, Clarke S, Díaz-Rubio E et al. Bevacizumab in combination with oxaliplatin-based chemotherapy as first-line therapy in metastatic colorectal cancer: a randomized phase III study. J Clin Oncol. $2008 \mathrm{Apr}$ 20;26(12):2013-9. 


\section{Case Reports in Oncology}

\begin{tabular}{l|l}
\hline Case Rep Oncol 2018;11:281-288 \\
\hline DOI: 10.1159/000489085 & $\begin{array}{l}\text { (c) 2018 The Author(s). Published by S. Karger AG, Basel } \\
\text { www.karger.com/cro }\end{array}$ \\
\hline
\end{tabular}


Fig. 1. Coronal and axial slices at the time of the initial diagnosis of metastatic pancreatic cancer on the left with biliary ductal dilation and a mass in the uncinate of the pancreas. 




Fig. 2. This scatterplot demonstrates the change in CA 19-9, a marker of the burden of pancreatic cancer, and the timeline of treatments from the time of initial presentation. 


\section{Case Reports in Oncology}

\begin{tabular}{l|l}
\hline Case Rep Oncol 2018;11:281-288 \\
\hline DOI: 10.1159/000489085 & $\begin{array}{l}\text { ( ) 2018 The Author(s). Published by S. Karger AG, Basel } \\
\text { www.karger.com/cro }\end{array}$ \\
\hline
\end{tabular}
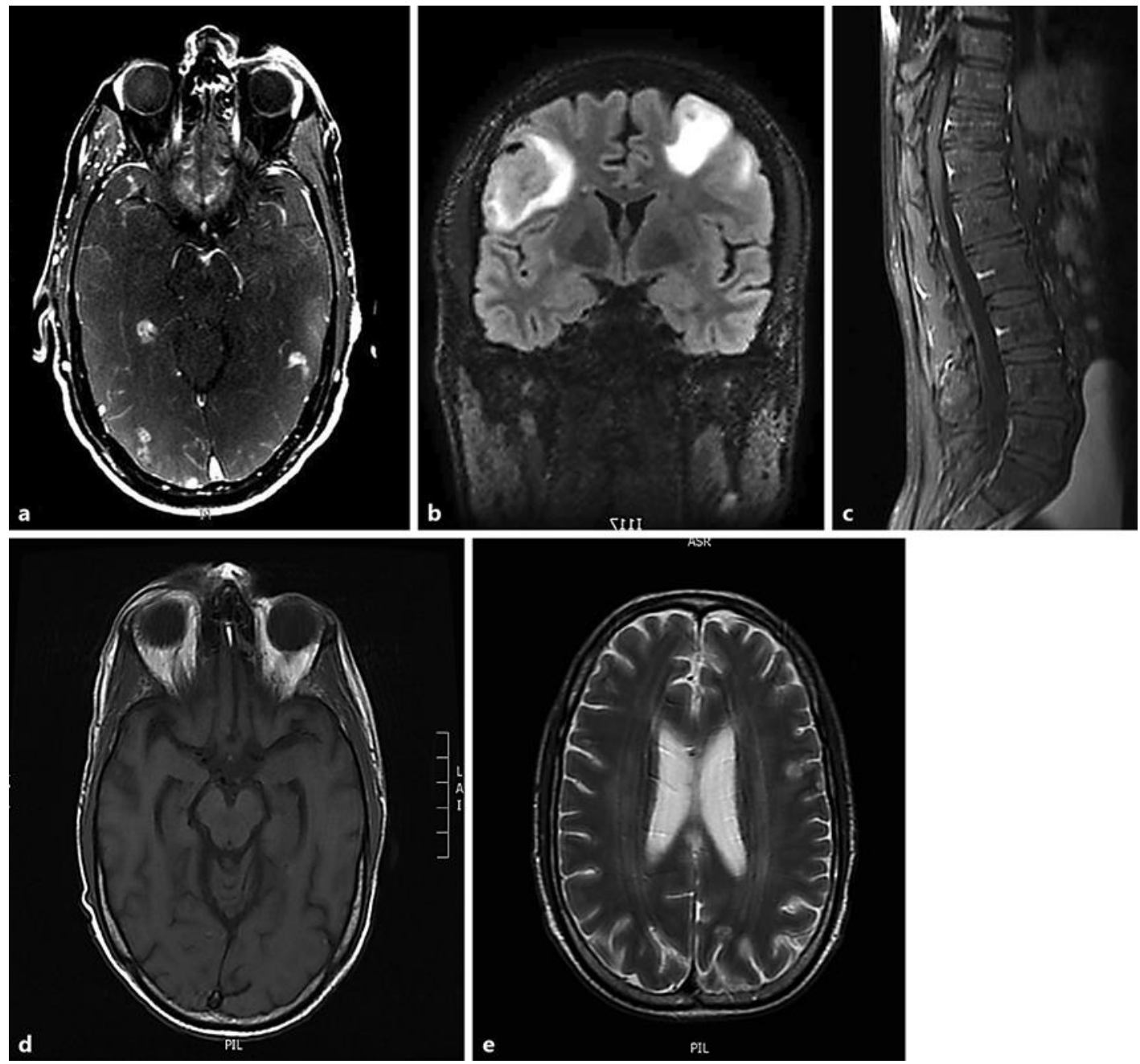

Fig. 3. Axial T1 (a), coronal FLAIR (b), and sagittal T1 (c) magnetic resonance images show metastases in the parenchyma, meninges, and spinal cord from initial diagnosis of central nervous system (CNS) involvement. Axial T1 (d) and T2 (e) show improved parenchymal disease about 8 months after initiation of CNStargeted therapy. 